\title{
Incidence and risk factors for tenofovir-associated nephrotoxicity among human immunodeficiency virus-infected patients in Korea
}

\author{
Jeong Eun Lee ${ }^{1}$, Shinwon Lee ${ }^{1}$, Sang Heon Song ${ }^{2}$, Ihm Soo Kwak ${ }^{2}$, and Sun Hee Lee ${ }^{1}$
}

Divisions of ${ }^{1}$ Infectious Disease and ${ }^{2}$ Nephrology, Department of Internal Medicine, Pusan National University Hospital, Busan, Korea

Received: December 14, 2016 Revised : March 31, 2017 Accepted: April 9, 2017

\section{Correspondence to}

Sun Hee Lee, M.D.

Division of Infectious Diseases, Department of Internal Medicine, Pusan National University Hospital, 179 Gudeok-ro, Seo-gu, Busan 49241, Korea

Tel: +82-51-240-7673

Fax: +82-51-247-3213

E-mail: zzanmery@gmail.com
Background/Aims: Little is known about tenofovir disoproxil fumarate (TDF)-induced nephrotoxicity in human immunodeficiency virus (HIV)-infected patients in Korea. The objective of this study was to evaluate the incidence and risk factors of TDF-associated nephrotoxicity among HIV-infected patients in Korea.

Methods: A single-center retrospective cohort study was conducted on HIVinfected patients in Korea. We included patients who had started TDF or abacavir (ABC)-based antiretroviral therapy (ART) between October 2006 and December 2014. Estimated glomerular filtration rate (eGFR) was estimated using the Chronic Kidney Disease-Epidemiology Collaboration equation. Renal dysfunction was defined as $>25 \%$ decrease of baseline eGFR. A propensity matched case-control study was conducted to compare renal dysfunction rates between the two groups. The risk factors of nephrotoxicity were analyzed by Cox regression analysis.

Results: A total of 210 HIV-infected patients were included in the study, of which, 108 were TDF-based ART group and 102 were ABC-based ART group. Renal dysfunction occurred in 16 patients (14.8\%) in the TDF group and 11 (10.8\%) in the $\mathrm{ABC}$ group. Incidence of renal dysfunction of TDF and $\mathrm{ABC}$ group was $9.66 \mathrm{per}$ 100 person-years (PYs) and 5.14 per 100 PYs, respectively $(p=0.176)$. In propensityscore-matched analysis, renal dysfunction rates were TDF 13.3\% versus ABC 13.3\% $(p>0.999)$. In multivariable analysis, Centers for Disease Control and Prevention clinical category $\mathrm{C}$ was a significant risk factor for renal dysfunction.

Conclusions: Approximately, 13\% of HIV-infected patients treated with TDF had renal dysfunction. Advanced stage of HIV infection was a significant risk factor for renal dysfunction.

Keywords: Tenofovir; HIV-infected patients; Nephrotoxicity; Centers for Disease Control and Prevention clinical category $\mathrm{C}$

\section{INTRODUCTION}

Tenofovir disoproxil fumarate (TDF) is one of the most widely used nucleotide reverse transcriptase inhibitors (NRTI) as a part of combination regimens for the treatment and prevention of human immunodeficiency virus (HIV) infection. TDF is available as either single agent or more frequently a component in several fixed dose combinations that is convenient. Although TDF is generally considered safe and well tolerated, its use has been associated with nephrotoxicity and a decrease in bone mineral density [1-3]. 
TDF can cause proximal renal tubular damage, in part due to potential mitochondrial toxicity, in severe cases leading to acute kidney injury and Fanconi's syndrome [4]. In addition, TDF nephrotoxicity can be manifested as distal tubular injury in the form of nephrogenic diabetes insipidus [1]. Renal wasting of phosphorus by damaged renal tubules can lead to osteomalacia. Previous studies suggest that older age, advanced HIV disease, low body weight, pre-existing renal impairment, and having comorbidities are risk factors of TDF-associated nephrotoxicity [5-9]. Concomitant use of a ritonavir or cobicistat with TDF was also suggested as a risk factor of renal dysfunction [10-12].

There are limited data on the incidence and risk factors for TDF-associated nephrotoxicity among the HIVinfected population in Korea. The objective of this study was to assess the incidence and risk factors of TDFassociated nephrotoxicity in HIV-infected patients in Korea. We also compared the incidence of nephrotoxicity among patients on TDF-based antiretroviral therapy (ART) with those on abacavir (ABC)-based ART.

\section{METHODS}

\section{Study population}

A retrospective study was conducted from January 2009 and December 2014 at a tertiary care hospital in Busan, South Korea. All HIV-infected patients who had started TDF (300 mg/day) or ABC (600 mg/day) were identified from computerized medical records from October 1 , 2006, to December 31, 2014. The patients who were $\geq 20$ years and with an estimated glomerular filtration rate (eGFR) of $\geq 60 \mathrm{~mL} / \mathrm{min} / 1.73 \mathrm{~m}^{2}$ with the Chronic Kidney Disease-Epidemiology Collaboration (CKD-EPI) equation at baseline were enrolled. Patients who met any of the following criteria were excluded: (1) both TDF- and ABC-based ART during study periods, (2) too short follow-up periods (12 weeks) and too limited data (no follow-up renal function test and viral load) to assess the effect of ART on renal function, (3) initiating ART in other clinics, (4) non-responder (no suppression of HIV viral load in the test performed 1 month after starting ART) or obviously non-compliant, and (5) administered with $300 \mathrm{mg}$ twice daily dose of ABC and Stribild (combined formulate of cobicistat, elvitegravir, emtricitabine and
TDF). ABC- or TDF-based ART were chosen by attending physicians according to their discretion at baseline. The observational periods were measured from the date of ART initiation to the earliest of the following dates: onset date of renal dysfunction, stop date of ABC or TDF if either of two drugs was switched to other drugs, date of the last follow-up if patients were lost to follow-up or transfer out, 120 weeks after ART initiation, or December 31, 2014.

\section{Assessment of renal dysfunction}

Renal dysfunction was defined as $>25 \%$ decrease of baseline eGFR. The baseline eGFR was calculated using the CKD-EPI equation: $\mathrm{eGFR}=141 \times \min (\mathrm{Scr} / \kappa, 1)^{\alpha} \times \max$ $(\mathrm{Scr} / \kappa, 1)^{-1.209} \times 0.993^{\text {Age }} \times 1.018$ (if female), where Scr is serum creatinine $(\mathrm{mg} / \mathrm{dL}), \kappa$ is 0.7 for females and 0.9 for males, $\alpha$ is -0.329 for females and -0.411 for males, min indicates the minimum of $\mathrm{Scr} / \mathrm{\kappa}$ or 1 , and max indicates the maximum of $\mathrm{Scr} / \mathrm{\kappa}$ or 1 [13].

\section{Data collection and definitions}

To identify the risk factors of renal dysfunction among HIV-infected patients receiving TDF- or ABC-based ART, we retrospectively collected demographic and clinical characteristic data from medical records including age, sex, body weight, body mass index (BMI), other medical conditions (concurrent use of nephrotoxic drugs, co-infection with hepatitis B and hepatitis C, hypertension, diabetes mellitus, and dyslipidemia), HIV viral load and cluster of differentiation $4^{+}\left(\mathrm{CD}_{4+}\right) \mathrm{T}$ cell count. AIDS defining illness was defined according to the 1993 AIDS Centers for Disease Control and Prevention (CDC) definition [14].

Hepatitis B virus (HBV) co-infection was defined as a positive HBs antigen test. Hepatitis C virus (HCV) coinfection was defined as a positive HCV antibody test. Diabetes mellitus, hypertension, dyslipidemia, and other underlying diseases were considered as comorbidities if documented in patients' medical records. Coadministration of nephrotoxic drugs were considered if patients had received aciclovir, valaciclovir, ganciclovir, aminoglycosides (gentamicin, amikacin, streptomycin), vancomycin, colistin, trimethoprim/sulfamethoxazole, and non-steroidal anti-inflammatory agents with ART. The concomitant ART used as boosted protease inhibitors (b-PIs) is ritonavir. 


\section{Statistical analysis}

Categorical variables were compared using Pearson chisquare test or Fisher exact test, whereas non-categorical variables were tested with Student $t$ test or the MannWhitney $U$ test. Incidence of nephrotoxicity was compared between TDF-based ART group and ABC-based ART group. We also performed a propensity score matched analyses to balance for differences in baseline characteristics between the two groups. The risk factors of TDF-associated nephrotoxicity were analyzed by multivariate proportional Cox regression analyses. All variables associated with renal dysfunction $(p<0.25)$ in univariate Cox models were assessed in multivariate models using stepwise forward selection. Statistical analyses were conducted using SPSS for Windows version 22.0 (IBM Co., Armonk, NY, USA).

\section{Ethics statement}

This retrospective study was approved by the Institutional Review Board of Pusan National University Hospital Clinical Trial Center, and the requirement informed consent was waived (E-2015062).

\section{RESULTS}

\section{Clinical characteristics}

Among 458 HIV-infected patients who had started TDF or ABC during the study period, 108 patients in the TDFbased ART group and 102 patients in the ABC-based ART group were enrolled after exclusion process. The baseline characteristics, comorbidities, and medications of the patients in both groups are presented in Table 1.

Both groups had a statistically similar predominance of male (TDF vs. ABC, $86.1 \%$ vs. $89.2 \%, p=0.495$ ), a relatively small body status (median weight, $63.48 \pm 12.00 \mathrm{~kg}$; median BMI, $22.1 \pm 3.57 \mathrm{~kg} / \mathrm{m}^{2}$ ), a high $\mathrm{CD}_{4}+\mathrm{T}$ cell count (TDF vs. ABC, $318.19 \pm 231.81 / \mu \mathrm{L}$ vs. $312.94 \pm 267.33 / \mu \mathrm{L}, p=$ 0.879), and high HIV RNA viral load (TDF vs. ABC, $3.95 \pm$ $1.82 \log _{10} / \mathrm{mL}$ vs. $\left.3.53 \pm 2.25 \log _{10} / \mathrm{mL}, p=0.137\right)$.

HBV co-infection rates (TDF vs. ABC, $16.7 \%$ vs. $3.9 \%$, $p=0.003$ ) and baseline eGFR (TDF vs. ABC, $107.63 \pm 15.50$ $\mathrm{mL} / \mathrm{min} / 1.73 \mathrm{~m}^{2}$ vs. $101.72 \pm 14.89 \mathrm{~mL} / \mathrm{min} / 1.73 \mathrm{~m}^{2}, p=$ 0.005) was significantly higher in the TDF-based ART group. Dyslipidemia was more frequent in the $\mathrm{ABC}$ based ART group (TDF vs. ABC, $0.9 \%$ vs. $7.8 \%, p=0.013$ ).
In the TDF-based ART group, the b-PIs and integrase inhibitors (INIs) were preferred than the nonboosted protease inhibitors (nb-PIs) and non-nucleoside reverse transcriptase inhibitors (NNRTIs) as concurrent ART (b-PIs 39.8\%, INIs 39.8\%, NNRTIs 14.8\%, nb-PIs 0.9\%), whereas in the ABC-based ART group, the b-PIs were the most favorite concurrent ART and the NNRTIs were preferred (b-PIs 54.9\%, NNRTIs $28.4 \%$, nb-PIs 9.8\%, INIs $6.9 \%$ ) (Table 1). The rate of concomitant administration of nephrotoxic drugs (TDF vs. ABC, $34.3 \%$ vs. $36.3 \%, p=$ 0.760 ) was similar between the two groups. Follow-up duration (weeks) was significantly longer in ABC-based ART group (TDF vs. ABC, $79.68 \pm 36.39$ vs. $109.01 \pm 26.96$, $p<0.001)$.

\section{Propensity-score-matched case control study}

The propensity-score-matched analysis was done to correct for differences in baseline characteristics between the two groups. To estimate the propensity score to receive TDF-based ART, age, body weight, BMI, baseline Scr, baseline eGFR, diabetes mellitus, dyslipidemia, HBV co-infection, CDC category, administration of nephrotoxic drug and follow-up duration were included. Seventy-five patients in TDF-based ART group were matched with 75 patients in ABC-based ART group with the closest propensity scores. Both groups were statistically similar, except follow-up duration (weeks) and the concurrent ART. Despite of the propensityscore-matched analysis, follow-up duration (weeks) was significantly longer in ABC-based ART group (TDF vs. $\mathrm{ABC}, 82.49 \pm 35.41$ vs. $105.59 \pm 30.62, p=0.004)$. In the TDF-based ART group, the INIs and b-PIs were preferred as concurrent ART (INIs 40.0\%, b-PIs 38.7\%, NNRTIs 14.7\%), whereas in the ABC-based ART group, the b-PIs were the most favorite concurrent ART and NNRTIs were preferred (b-PIs 54.7\%, NNRTIs 30.7\%, nbPIs 8.0\%, INIs 6.7\%) (Table 2).

\section{The incidence of renal dysfunction}

Renal dysfunction occurred in 16 patients (14.8\%) in the TDF-based ART group and 11 (10.8\%) in the ABC-based ART group, with an estimated incidence of 9.66 and 5.14 per 100 person-years (PYs), respectively. The median time from initiation of ART to occurrence of renal dysfunction was 30.15 weeks (range, o to 110) for the TDFbased arm and 50.62 weeks (range, 5 to 120 ) for $A B C$ arm. 
Table 1. Baseline demographics and laboratory data of patients who received TDF- or ABC-based ART $(n=210)$

\begin{tabular}{|c|c|c|c|}
\hline Variable & $\operatorname{TDF}(\mathrm{n}=108)$ & $\operatorname{ABC}(\mathrm{n}=102)$ & pvalue \\
\hline Male sex & $93(86.1)$ & $91(89.2)$ & 0.495 \\
\hline Age, yr & $46.15 \pm 13.16$ & $44.75 \pm 12.94$ & 0.437 \\
\hline Body weight, kg & $62.21 \pm 12.20$ & $64.83 \pm 11.69$ & 0.114 \\
\hline $\mathrm{BMI}, \mathrm{kg} / \mathrm{m}^{2}$ & $21.88 \pm 3.77$ & $22.33 \pm 3.34$ & 0.359 \\
\hline $\mathrm{CD}_{4}$ count, $/ \mu \mathrm{L}$ & $318.19 \pm 231.81$ & $312.94 \pm 267.33$ & 0.879 \\
\hline HIV RNA virus, $\log / \mathrm{mL}$ & $3.95 \pm 1.82$ & $3.53 \pm 2.25$ & 0.137 \\
\hline Hepatitis B & $18(16.7)$ & $4(3.9)$ & 0.003 \\
\hline Hepatitis C & $7(6.5)$ & $7(6.9)$ & 0.912 \\
\hline Hypertension & $16(14.8)$ & $15(14.7)$ & 0.982 \\
\hline Diabetes mellitus & $8(7.4)$ & $12(11.8)$ & 0.282 \\
\hline Dyslipidemia & $1(0.9)$ & $8(7.8)$ & 0.013 \\
\hline Nephrotoxic drug & $37(34 \cdot 3)$ & $37(36.3)$ & 0.760 \\
\hline CDC clinical category C & $24(22.2)$ & $16(15 \cdot 7)$ & 0.228 \\
\hline Serum creatinine, mg/dL & $0.78 \pm 0.17$ & $0.85 \pm 0.18$ & 0.001 \\
\hline Baseline eGFR, $\mathrm{mL} / \mathrm{min} / 1.73 \mathrm{~m}^{2}$ & $107.63 \pm 15.50$ & $101.72 \pm 14.89$ & 0.005 \\
\hline Follow-up duration, wk & $79.68 \pm 36.39$ & $109.01 \pm 26.96$ & $<0.001$ \\
\hline Marital status, married & $48(45 \cdot 3)$ & $48(47 \cdot 1)$ & 0.797 \\
\hline \multicolumn{4}{|l|}{ Mode of transmission } \\
\hline Heterosexual & $45(42.9)$ & $52(52.5)$ & 0.167 \\
\hline Homosexual & $59(56.2)$ & $45(45 \cdot 5)$ & 0.125 \\
\hline Others & $1(1)$ & $2(2)$ & 0.527 \\
\hline \multicolumn{4}{|l|}{ Concurrent ART } \\
\hline b-PIs & $43(39.8)$ & $56(54 \cdot 9)$ & 0.029 \\
\hline $\mathrm{nb}-\mathrm{PIs}$ & $1(0.9)$ & $10(9.8)$ & 0.004 \\
\hline NNRTIs & $16(14.8)$ & $29(28.4)$ & 0.016 \\
\hline INIs & $43(39.8)$ & $7(6.9)$ & $<0.001$ \\
\hline $\operatorname{Mix}^{\mathrm{a}}$ & $5(4 \cdot 6)$ & 0 & 0.028 \\
\hline
\end{tabular}

Values are presented as number (\%) or mean \pm SD.

TDF, tenofovir; ABC, abacavir; ART, antiretroviral therapy; BMI, body mass index; CD4, cluster of differentiation 4; HIV, human immunodeficiency virus; CDC, Centers for Disease Control and Prevention; eGFR, estimated glomerular filtration rate; b-PI, boosted protease inhibitor; nb-PI, nonboosted protease inhibitor; NNRTI, non-nucleoside reverse transcriptase inhibitor; INI, integrase inhibitor.

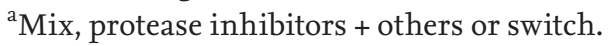

The total observation period was 447.2 patient-years (median, 839 days; interquartile range [IQR], 357 to 1,137) for the TDF arm and 901.7 patient-years (median, 1,119 days; IQR, 660.5 to 1,509) for the ABC arm.

Fig. 1 shows the time from ART initiation to $25 \%$ decrease in eGFR by the Kaplan-Meier method in the two groups. The estimated incidence of renal dysfunction in TDF- and ABC-based ART groups was 9.66 per 100 PYs and 5.14 PYs, respectively. In surviving analysis, ABC- based ART group showed lower renal dysfunction prevalence, but the difference across groups did not reach statistical significance $(p=0.176)$ (Fig. 1A). In propensity-score-matched analysis, renal dysfunction occurred in 10 patients $(13.3 \%)$ in the TDF-based ART group and $10(13.3 \%)$ in the ABC-based ART group, with an estimated incidence of 8.41 and 6.57 per 100 PYs, respectively ( $p$ $>0.999$ ) (Table 2). Fig. 1B shows the time from ART initiation to $>25 \%$ decrease in eGFR by the Kaplan-Meier 
Table 2. Baseline demographics and laboratory data and comparison of implications of ART on renal function of propensity score-matched patients who received TDF- and ABC-based ART (n=150)

\begin{tabular}{|c|c|c|c|}
\hline Variable & $\operatorname{TDF}(\mathrm{n}=75)$ & $\mathrm{ABC}(\mathrm{n}=75)$ & pvalue \\
\hline Male sex & $63(86.0)$ & $66(88.0)$ & 0.664 \\
\hline Age, yr & $45.19 \pm 14.34$ & $45 \cdot 32 \pm 13 \cdot 71$ & 0.956 \\
\hline Body weight, kg & $63.63 \pm 12.94$ & $63.93 \pm 11.00$ & 0.880 \\
\hline $\mathrm{BMI}, \mathrm{kg} / \mathrm{m}^{2}$ & $22.13 \pm 3.92$ & $22.08 \pm 3.14$ & 0.936 \\
\hline $\mathrm{CD}_{4}$ count, $/ \mu \mathrm{L}$ & $317.95 \pm 230.75$ & $292.95 \pm 242.17$ & 0.518 \\
\hline HIV RNA virus, $\log / \mathrm{mL}$ & $3.96 \pm 1.77$ & $3.69 \pm 2.26$ & 0.423 \\
\hline Hepatitis B & $1(1.3)$ & $4(5 \cdot 3)$ & 0.367 \\
\hline Hepatitis C & $5(6.7)$ & $7(9 \cdot 3)$ & 0.765 \\
\hline Hypertension & $11(14 \cdot 7)$ & $12(16.0)$ & $>0.999$ \\
\hline Diabetes mellitus & $6(8.0)$ & $8(10.7)$ & 0.780 \\
\hline Dyslipidemia & $1(1.3)$ & $5(6.7)$ & 0.209 \\
\hline Nephrotoxic drug & $26(34.7)$ & $27(36.0)$ & $>0.999$ \\
\hline CDC clinical category C & $15(20.0)$ & $15(20.0)$ & $>0.999$ \\
\hline Serum creatinine, mg/dL & $0.81 \pm 0.17$ & $0.83 \pm 0.16$ & 0.440 \\
\hline Baseline eGFR, mL/min/1.73 $\mathrm{m}^{2}$ & $106.41 \pm 15.85$ & $103.96 \pm 15.02$ & 0.333 \\
\hline Follow-up duration, wk & $82.49 \pm 35.41$ & $105.59 \pm 30.62$ & 0.004 \\
\hline Marital status, married & $32(43.8)$ & $37(49 \cdot 3)$ & 0.515 \\
\hline \multicolumn{4}{|l|}{ Mode of transmission } \\
\hline Heterosexual & $33(45.8)$ & $39(53 \cdot 4)$ & 0.408 \\
\hline Homosexual & $38(52.8)$ & $32(43.8)$ & 0.320 \\
\hline Others & $1(1.4)$ & $2(2.7)$ & $>0.999$ \\
\hline \multicolumn{4}{|l|}{ Concurrent ART } \\
\hline b-PIs & $29(38.7)$ & $41(54 \cdot 7)$ & 0.050 \\
\hline nb-PIs & o & $6(8.0)$ & 0.012 \\
\hline NNRTIs & $11(14.7)$ & $23(30.7)$ & 0.031 \\
\hline INIs & $30(40.0)$ & $5(6.7)$ & $<0.001$ \\
\hline $\operatorname{Mix}^{\mathrm{a}}$ & $5(6.7)$ & $\mathrm{o}(0.0)$ & 0.058 \\
\hline eGFR of the end of study, $\mathrm{mL} / \mathrm{min} / 1.73 \mathrm{~m}^{2}$ & $98.91 \pm 21.52$ & $100.45 \pm 16.08$ & 0.613 \\
\hline Renal dysfunction & $10(13 \cdot 3)$ & $10(13 \cdot 3)$ & $>0.999$ \\
\hline
\end{tabular}

Values are presented as number (\%) or mean \pm SD.

ART, antiretroviral therapy; TDF, tenofovir; ABC, abacavir; BMI, body mass index; CD4, cluster of differentiation 4; HIV, human immunodeficiency virus; CDC, Centers for Disease Control and Prevention; eGFR, estimated glomerular filtration rate; b-PI, boosted protease inhibitor; nb-PI, nonboosted protease inhibitor; NNRTI, non-nucleoside reverse transcriptase inhibitor; INI, integrase inhibitor.

${ }^{\mathrm{a}}$ Mix, protease inhibitors + others or switch.

analyses in the two groups $(p=0.723)$.

\section{Risk factors for TDF-associated nephrotoxicity after propensity-score matching}

In the univariate analysis, nephrotoxic drug (hazard ratio [HR], 3.68; 95\% confidence interval [CI], 1.47 to 9.23; $p=0.005)$, hypertension (HR, 2.76; 95\% CI, 1.06 to 7.19; $p$ = 0.037), and CDC clinical category C (adjusted HR, 5.83; $95 \%$ CI, 2.41 to 14.09 ; $p<0.001$ ) were significantly associated with the renal dysfunction. However, TDF use was not associated with the renal dysfunction $(p=0.723)$ (Table 3). 

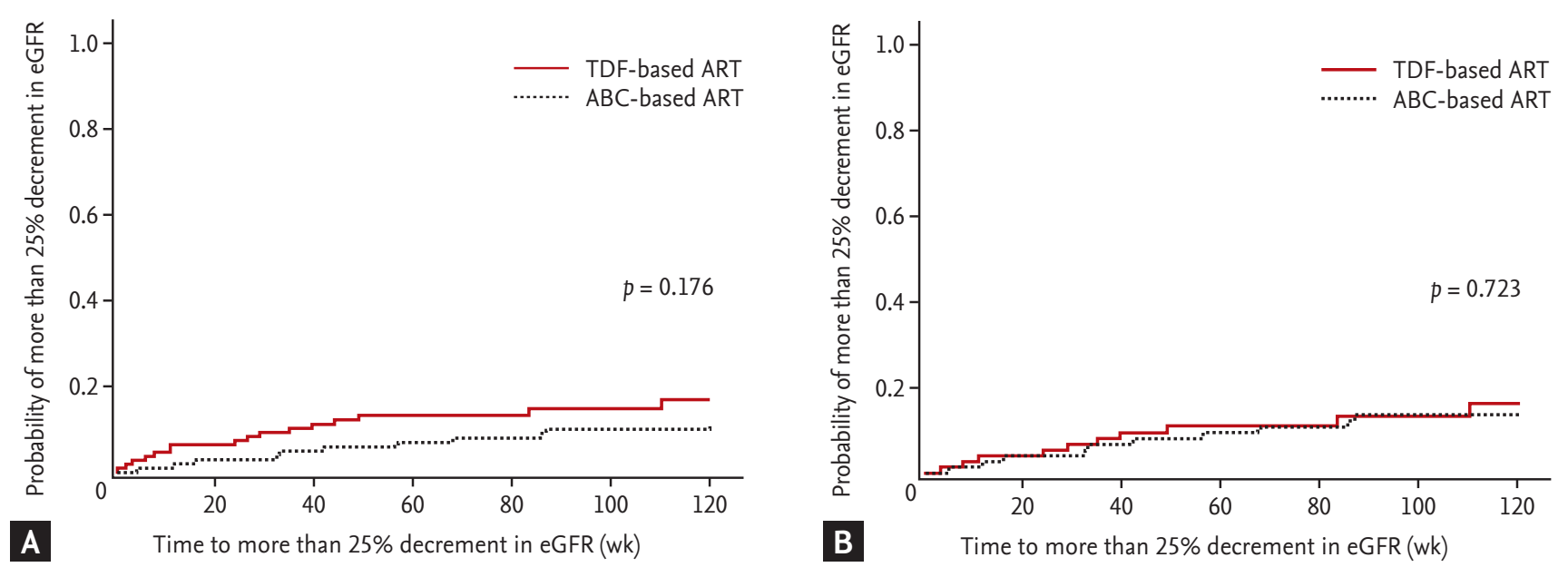

Figure 1. Kaplan-Meier curve showing the time to renal dysfunction in patients with tenofovir (TDF)- or abacavir (ABC)-based antiretroviral therapy (ART). (A) Before propensity score matching. (B) After propensity score matching. eGFR, estimated glomerular filtration rate.

In multivariate analysis, $\mathrm{CDC}$ clinical category $\mathrm{C}(\mathrm{HR}$, 8.09; $95 \%$ CI, 2.46 to 26.57 ; $p=0.001$ ) was the only significant risk factor for renal dysfunction (Table 3 ).

\section{DISCUSSION}

In this study, the incidence of renal dysfunction, defined as $>25 \%$ decrement of eGFR using CKD-EPI from the baseline was 9.66 per 100 PYs in HIV-infected patients who started TDF-based ART. Despite the possibility of some differences in the incidence or prevalence of TDF-associated nephrotoxicity according to the measuring methods of eGFR and definition of TDF-associated nephrotoxicity, the results were broadly in line with those of other studies $[6,9,15,16]$. A retrospective study conducted in Korea showed that the incidence of acute Scr elevation $>1.5 \mathrm{mg} / \mathrm{dL}$ regardless of baseline Scr level among patients treated with TDF-containing regimens was 2.8 per 100 PYs [17].

In our study, TDF- and ABC-based ART groups showed some important differences in baseline characteristics. First, there was a difference in baseline eGFR between two groups possibly because physicians frequently prescribed ABC-based ART than TDF-based ART to patients with lower renal function or several risk factors for renal dysfunction. Second, TDF-based ART was more frequently used in the HIV and HBV co-infected patients than ABC-based ART, since anti-retrovi- ral agent which is effective against both HIV and HBV (for example, tenofovir, lamivudine, emtricitabine) can also prevent the occurrence of clinically significant liver disease by directly inhibiting the replication of HBV [18]. Third, the duration of the use of ABC-based ART was longer than that of ABC-based ART because of the difference of the timing of the introduction of $A B C$ (in 2002) and TDF (in 2012).

The propensity-score-matched analysis was conducted to adjust for group-wise differences in baseline characteristics. After propensity-scores were matched, renal dysfunction rate was similar between the two groups (TDF 13.3\% vs. ABC 13.3\%); and hypertension, co-administration of nephrotoxic drugs and CDC clinical category C were significantly associated with renal dysfunction, but TDF use, older age and ritonavir b-PIs use were not associated in univariate analysis. There was no statistically significant difference in the age distribution between before and after 40 years of age (HR, 2.44; 95\% CI, 0.81 to $7.28 ; p=0.112$ ) or before and after 60 years of age (HR, 1.54; 95\% CI, 0.56 to $4.24 ; p=0.404$ ). In multivariate analyses, $\mathrm{CDC}$ clinical category $\mathrm{C}$ was only significantly associated with the renal dysfunction. Consistent with previous studies, advanced stage was a significant risk factor for TDF-associated nephrotoxicity in our study.

After adjusting for variables, we expected a higher rate of renal dysfunction in TDF-based ART group than ABC-based ART group. However, the difference in rate of renal dysfunction between the two groups was de- 
Table 3. Univariate and multivariate analysis for factors associated with nephrotoxicity after propensity-score matching

\begin{tabular}{|c|c|c|c|c|}
\hline \multirow{2}{*}{ Variable } & \multicolumn{2}{|c|}{ Univariate analysis } & \multicolumn{2}{|c|}{ Multivariate analysis } \\
\hline & $\operatorname{HR}(95 \% \mathrm{CI})$ & $p$ value & $\operatorname{HR}(95 \% \mathrm{CI})$ & $p$ value \\
\hline \multicolumn{5}{|l|}{ Backbone NRTIs } \\
\hline $\mathrm{ABC}$ & 1 & - & - & - \\
\hline TDF & $1.17(0.49-2.83)$ & 0.723 & - & - \\
\hline \multicolumn{5}{|l|}{ Sex } \\
\hline Male & 1 & - & - & - \\
\hline Female & $0.31(0.04-2.31)$ & 0.252 & - & - \\
\hline \multicolumn{5}{|l|}{ Age, yr } \\
\hline$\leq 30$ & 1 & - & 1 & - \\
\hline $31-50$ & $3.04(0.38-24.30)$ & 0.295 & $1.55(0.18-13.31)$ & 0.689 \\
\hline$>50$ & $5.73(0.74-44.37)$ & 0.095 & $3.36(0.39-29.08)$ & 0.272 \\
\hline \multicolumn{5}{|l|}{ BMI, $\mathrm{kg} / \mathrm{m}^{2}$} \\
\hline$<18.5$ & 1 & - & - & - \\
\hline $18.5-24.9$ & $0.64(0.21-1.98)$ & 0.441 & - & - \\
\hline$\geq 25$ & $0.51(0.11-2.22)$ & 0.377 & - & - \\
\hline \multicolumn{5}{|l|}{$\mathrm{CD} 4$ count, $/ \mu \mathrm{L}$} \\
\hline$>500$ & 1 & - & - & - \\
\hline $201-500$ & $0.74(0.18-3.10)$ & 0.681 & - & - \\
\hline$\leq 200$ & $2.21(0.62-7.83)$ & 0.219 & - & - \\
\hline \multicolumn{5}{|l|}{ HIV RNA virus, $\log / \mathrm{mL}$} \\
\hline Undetectable & 1 & - & 1 & - \\
\hline $\log 0-5$ & $2.23(0.47-10.49)$ & 0.312 & $2.82(0.56-14.26)$ & 0.210 \\
\hline$>\log 5$ & $3.94(0.86-17.98)$ & 0.077 & $1.74(0.34-8.93)$ & 0.507 \\
\hline \multicolumn{5}{|l|}{ Concurrent ART } \\
\hline b-PIs & 1 & - & 1 & - \\
\hline Others $^{\mathrm{a}}$ & $0.56(0.23-1.38)$ & 0.207 & $0.48(0.18-1.25)$ & 0.131 \\
\hline Hepatitis B vs. none & $1.49(0.20-11.15)$ & 0.697 & - & - \\
\hline Hepatitis C vs. none & $1.38(0.32-5.97)$ & 0.664 & - & - \\
\hline Hypertension vs. none & $2.76(1.06-7.19)$ & 0.037 & $2.12(0.52-8.14)$ & 0.293 \\
\hline Diabetes mellitus vs. none & $2.87(0.96-8.57)$ & 0.060 & $1.81(0.40-8.20)$ & 0.442 \\
\hline Dyslipidemia vs. none & $1.20(0.16-8.98)$ & 0.858 & - & - \\
\hline Nephrotoxic drug vs. none & $3.68(1.47-9.23)$ & 0.005 & $1.14(0.35-3.73)$ & 0.825 \\
\hline CDC, class C vs. A or B & $5.83(2.41-14.09)$ & $<0.001$ & $8.09(2.46-26.57)$ & 0.001 \\
\hline
\end{tabular}

HR, hazard ratio; CI, confidence interval; NRTI, nucleotide reverse transcriptase inhibitor; ABC, abacavir; TDF, tenofovir; BMI, body mass index; CD4, cluster of differentiation 4; HIV, human immunodeficiency virus; ART, antiretroviral therapy; b-PI, boosted protease inhibitor; CDC, Centers for Disease Control and Prevention.

${ }^{\mathrm{a}}$ Others, concurrent ART except the b-PIs.

creased, possibly due to exclusion of a relatively large number of patients in serious condition (category $\mathrm{C}$ ) in TDF-based ART group than ABC-based ART group by propensity score matching (category C: before matching, TDF $22.2 \%$ vs. ABC $15.7 \%$; after matching, TDF $20.0 \%$ vs.
ABC 20.0\%). The mean body weight was slightly lower in the TDF-based ART group before matching and the mean body weight was more similar after matching, without significance (before matching, TDF $62.21 \pm 12.20$ kg vs. ABC $64.83 \pm 11.69 \mathrm{~kg}, \mathrm{p}=0.114$; after matching, TDF 
$63.63 \pm 12.94 \mathrm{~kg}$ vs. ABC $63.93 \pm 11.00 \mathrm{~kg}, p=0.880)$. Low body weight is a well-known risk factor for renal failure when using TDF [5-7]. Exclusion of low body weight patients in the TDF-based ART group from the matching could have an important effect. Therefore, use of TDF as the NRTIs back bone does not significantly increase the risk of renal dysfunction in cases with good baseline renal function and HIV/AIDS not manifesting as a serious condition.

This study has several limitations. First, this study is a retrospective observational study, so we cannot rule out the presence of unmeasured confounding. Second, there were a differences in the timing of the introduction of the ABC and TDF in Korea. Third, it was difficult to adjust for significant group-wise difference in concurrent ART use; hence, it was difficult to assess nephrotoxicity due to concurrent ART use. Fourth, HIV-infected patients with relatively low eGFR were prescribed more ABC-based ART than TDF-based ART, so there was a difference in baseline eGFR between the two groups. Fifth, because the maximum follow-up duration is 120 weeks, it is necessary to observe a rate of change in nephrotoxicity when long-term follow-up. Sixth, CDC clinical category was a larger confound factor than TDF use because there was a lot of HIV-infected patients with serious clinical condition in our study. Finally, several studies showed useful screening markers for tubular cell damage because of TDF, such as urinary b2M, proteinuria, glycosuria and cystatin C, but we did not evaluate these markers.

In conclusion, HIV-infected patients with TDF use showed a tendency for more frequent occurrence of renal dysfunction than those with $\mathrm{ABC}$ use. Advanced stage of HIV infection was an important risk factor for renal dysfunction in HIV-infected patients receiving ART in Korea.

\section{KEY MESSAGE}

1. Tenofovir disoproxil fumarate use was not associated with nephrotoxicity in human immunodeficiency virus-infected Korean patients if baseline renal function was normal.

2. The Centers for Disease Control and Prevention category $\mathrm{C}$ was identified as an independent risk factor associated with nephrotoxicity.

\section{Conflict of interest}

No potential conflict of interest relevant to this article was reported.

\section{Acknowledgments}

This work was supported by a 2-year Research Grant of Pusan National University.

\section{REFERENCES}

1. Karras A, Lafaurie M, Furco A, et al. Tenofovir-related nephrotoxicity in human immunodeficiency virus-infected patients: three cases of renal failure, Fanconi syndrome, and nephrogenic diabetes insipidus. Clin Infect Dis 2003;36:1070-1073.

2. Zimmermann AE, Pizzoferrato T, Bedford J, Morris A, Hoffman R, Braden G. Tenofovir-associated acute and chronic kidney disease: a case of multiple drug interactions. Clin Infect Dis 2006;42:283-290.

3. Perrot S, Aslangul E, Szwebel T, Caillat-Vigneron N, Le Jeunne C. Bone pain due to fractures revealing osteomalacia related to tenofovir-induced proximal renal tubular dysfunction in a human immunodeficiency virus-infected patient. J Clin Rheumatol 2009;15:72-74.

4. Perazella MA. Tenofovir-induced kidney disease: an acquired renal tubular mitochondriopathy. Kidney Int 2010;78:1060-1063.

5. Gervasoni C, Meraviglia P, Landonio S, et al. Low body weight in females is a risk factor for increased tenofovir exposure and drug-related adverse events. PLoS One 2013;8:e80242.

6. Nishijima T, Gatanaga H, Komatsu H, et al. Renal function declines more in tenofovir- than abacavir-based antiretroviral therapy in low-body weight treatment-naïve patients with HIV infection. PLoS One 2012;7:e29977.

7. Nishijima T, Komatsu H, Gatanaga H, et al. Impact of small body weight on tenofovir-associated renal dysfunction in HIV-infected patients: a retrospective cohort study of Japanese patients. PLoS One 2011;6:e22661.

8. Marchewka Z, Szymczak A, Knysz B. Comorbidities as risk factors of chronic kidney disease in HIV-infected persons. Postepy Hig Med Dosw (Online) 2015;69:13641370.

9. Pinto Neto LF, Bassetti BR, Fraga IH, Oliveira Santos CR, Ximenes PD, Miranda AE. Nephrotoxicity during tenofo- 
vir treatment: a three-year follow-up study in a Brazilian reference clinic. Braz J Infect Dis 2016;20:14-18.

10. Kearney BP, Mathias A, Mittan A, Sayre J, Ebrahimi R, Cheng AK. Pharmacokinetics and safety of tenofovir disoproxil fumarate on coadministration with lopinavir/ ritonavir. J Acquir Immune Defic Syndr 2006;43:278-283.

11. Gallant JE, Moore RD. Renal function with use of a tenofovir-containing initial antiretroviral regimen. AIDS 2009;23:1971-1975.

12. Goicoechea M, Liu S, Best B, et al. Greater tenofovir-associated renal function decline with protease inhibitor-based versus nonnucleoside reverse-transcriptase inhibitor-based therapy. J Infect Dis 2008;197:102-108.

13. Ji M, Lee YH, Hur M, et al. Comparing results of five glomerular filtration rate-estimating equations in the Korean General Population: MDRD study, revised Lund-Malmo, and three CKD-EPI equations. Ann Lab Med 2016;36:521-528.

14. Centers for Disease Control and Prevention (CDC). Update: impact of the expanded AIDS surveillance case definition for adolescents and adults on case reporting.
United States, 1993. MMWR Morb Mortal Wkly Rep 1994;43:160-161, 167-170.

15. Rodriguez-Novoa S, Labarga P, Soriano V, et al. Predictors of kidney tubular dysfunction in HIV-infected patients treated with tenofovir: a pharmacogenetic study. Clin Infect Dis 2009;48:e108-e116.

16. Cooper RD, Wiebe N, Smith N, Keiser P, Naicker S, Tonelli M. Systematic review and meta-analysis: renal safety of tenofovir disoproxil fumarate in HIV-infected patients. Clin Infect Dis 2010;51:496-505.

17. Jin S, Kim MH, Park JH, et al. The incidence and clinical characteristics of acute serum creatinine elevation more than $1.5 \mathrm{mg} / \mathrm{dl}$ among the patients treated with tenofovir/emtricitabine-containing HAART regimens. Infect Chemother 2015;47:239-246.

18. Rotheram-Borus MJ, Swendeman D, Chovnick G. The past, present, and future of HIV prevention: integrating behavioral, biomedical, and structural intervention strategies for the next generation of HIV prevention. Annu Rev Clin Psychol 2009;5:143-167. 